\title{
Semiconductor Sensor Layer in SAW Gas Sensors Configuration
}

\author{
T. HEJCZYK ${ }^{a}$, M. URBAŃCZYK ${ }^{b}$ AND W.P. JAKUBIK ${ }^{c}$ \\ ${ }^{a}$ WASKO S.A., Berbeckiego 6, 44-100 Gliwice, Poland \\ ${ }^{b}$ Faculty of Electrical Engineering, Silesian University of Technology, Krzywoustego 2, 44-100 Gliwice, Poland \\ ${ }^{c}$ Institute of Physics, Silesian University of Technology, Krzywoustego 2, 44-100 Gliwice, Poland \\ A Rayleigh acoustic wave travelling on the surface of a semi-infinite piezoelectric medium may be changed \\ by interaction with carriers and diffused gas in an adjacent semiconductor. The configuration, which uses a \\ thin semiconductor film supported by a catalytic layer $(\mathrm{Pd})$, is described in detail and the theoretical results of \\ gas-sensor layer interaction are presented.
}

PACS numbers: 77.65.Dq, 68.35.Iv, 07.07.Df

\section{Introduction}

A very interesting feature of the surface acoustic wave (SAW) sensors is the fact that the layered sensor structure on a piezoelectric substrate provides new possibilities for detecting gas in a SAW sensor system by using the acoustoelectric coupling between the surface wave and the free charges in semiconductor sensor layer. Using a surface acoustic wave surface with the dual delay line and an adequately chosen active layer creates possibilities for designing a sensor with high sensitivity and good temperature stability.

SAW gas sensors are attractive because of their remarkable sensitivity due to changes of the boundary conditions (mechanical and especially electrical in acoustoelectric effect) of the propagating wave, introduced by interaction of an active thin sensor film with specific gas molecules. This unusual sensitivity results from the simple fact that most of the acoustic wave energy is concentrated near the crystal surface within approximately one or two wavelengths. The surface wave is in its first approximation highly sensitive to any changes of the physical or chemical properties of the thin active layer previously placed on the crystal surface [1-3].

As has been mentioned earlier, any change in the physical or chemical properties of the thin active layer placed on a piezoelectric surface can affect SAW propagation. However, from a practical point of view, only the following two effects are potentially significant; namely, a change in the mass of the layer and a change of its electrical conductivity, which cause a significant change in the velocity and attenuation of SAW. These two effects occur simultaneously in the interaction time of an active film with a specific gas concentration. For instance, in thin phtalocyanine films as organic semiconductors both of these exist, but the electric effect is much greater (several times, depending on the gas concentration) $[4,5]$.

For sensor construction, optimization is important in order to get an analytical model of the SAW sensor. In this paper we give some theoretical information on the acoustoelectric effect in the piezoelectric acoustic delay line and semiconducting sensor layer configuration. This will be the starting point to construct a multilayer analytical model, because the sensor layer with absorbed gas molecules may be treated as the sum of the partial layers with a different electrical conductivity.

\section{Schematic diagram of the SAW sensor}

The experimental setup is based on frequency changes in a surface acoustic wave dual delay system, which is nowadays well known $[5,6]$. Investigation of thin film structures of $\mathrm{WO}_{3}$ was precisely described in work [4]. This system is schematically shown in Fig. 1.

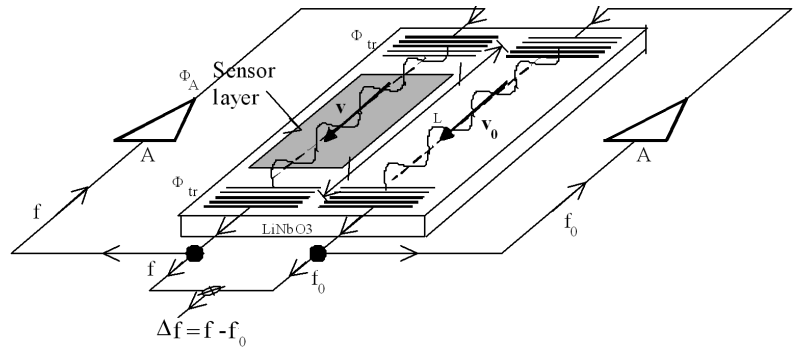

Fig. 1. SAW dual delay-line oscillator system. SAW dual line configuration with a sensor thin layer structure in a measuring line. $\mathrm{A}-$ amplifier [6].

Systems with the Rayleigh SAW have been applied in the construction of detectors of toxic gases for a long 
time. For this purpose, on a piezoelectric substrate (e.g. $\mathrm{LiNbO}_{3}$ ) two identical circuits are formed to facilitate the propagation on the surface wave excited by means of interdigital transducers. Next, by thermal sublimation in a vacuum or radio frequency (RF) sputtering in one of the paths a thin layer of an organic or inorganic semiconductor is formed. If its thickness is not exceeding $1 \%$ of the length of the propagating surface wave, then we can accept that the acoustic wave in this system is a Rayleigh type. The free path of a crystal serves as a reference, permitting easy measurements of the obtained differential frequency, $\Delta f$, and can compensate small variations of surrounding temperature and air pressure. Both delay lines are placed in the feedback loop oscillator circuits and the response to the particular gas of the active layer is detected as a change of the differential frequency, $\Delta f$, i.e. the difference between the two oscillator frequencies $f$ and $f_{0}$.

\section{Acoustoelectric effect in the piezoelectric- semiconductor layer}

In the case of small disturbances, both mass and electric load mentioned above may be considered separately, assuming that the total effect of a relative change of wave vector $\Delta k / k_{0}$ and the velocity of propagation $\Delta v / v_{0}$ is the sum of both these component disturbances [7, 8]:

$$
\frac{\Delta k}{k_{0}} \approx\left(\frac{\Delta k}{k_{0}}\right)_{m}+\left(\frac{\Delta k}{k_{0}}\right)_{\sigma} .
$$

Making use of the theory of disturbances, the contribution of each of these effects can be determined theoretically. From the physical point of view it seems to be essential that the influence of each effect (mass and electrical) should be considered separately from its reaction to the interaction of the layer with gas. In the case of an electric effect we are assuming that the mass of the layer $m=0$. The electric "load" is resulting from the effect of the interaction of the electric potential associated with the surface wave with mobile charge carriers in the layer.

\subsection{Electrical surface perturbations}

In these problems the mechanical boundary conditions are unperturbed. If the electrical boundary conditions are perturbed only at the upper surface $y=0$, the perturbation formula is then

$$
(\Delta k)_{\sigma} \approx \frac{\omega\left[\varphi^{\prime}(0) D_{y}^{*}(0)-\varphi^{*}(0) D_{y}^{\prime}(0)\right]}{4 P},
$$

where index $\sigma$ is referred to the change of wave number by disturbances, electrical surface perturbations.

In these problems the mechanical boundary conditions $(T=0)$ are unperturbed. Unperturbed electrical boundary conditions are not usually either short-circuit $(\varphi(0)=0)$ or open-circuit $\left(D_{y}(0)=0\right)$ and it is not clear whether the potential or the electrical displacement should be approximated by its unperturbed value. The best agreement between perturbation theory and exact numerical calculations is obtained by using the so-called weak coupling approximation in which the stress field $T$ is assumed to be unchanged by the perturbation [1].

To illustrate the method, a perturbation formula will be derived for the important practical case of the piezoelectric Rayleigh wave [3].

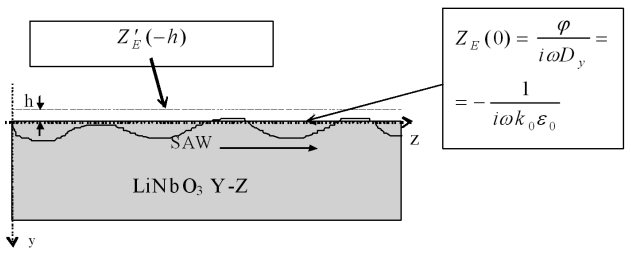

Fig. 2. Electrical surface perturbations. The perturbation of the SAW velocity is resulting from changes in the electric conductivity of the layer, $\sigma_{\mathrm{s}}$, due to the interaction of the chemically active layers with gas.

In doing this it is most convenient to express the electrical boundary conditions in terms of the electrical surface impedance. This is an impedance per unit area

$$
Z_{E}(0)=\left(\frac{\varphi}{\mathrm{i} \omega D_{y}}\right)_{y=0} .
$$

It will be assumed that unperturbed problem has free electrical boundary conditions at the substrate surface, that is, the region above the substrate $(y<0$ in Fig. 2$)$ is vacuum and extends to $y \rightarrow \infty$.

The space-charge potential satisfying the general form of Laplace's equation [2]:

$$
\nabla \varepsilon \cdot \nabla \varphi_{\mathrm{S}}=0 .
$$

For this region Laplace equation reduces to the form

$$
\nabla^{2} \Phi=0 \text {. }
$$

The unperturbed potential function is therefore

$$
\Phi=\Phi_{\mathrm{R}}(y) \mathrm{e}^{-\mathrm{i} k z}=\mathrm{e}^{k y} \mathrm{e}^{-\mathrm{i} k z}, \quad y<0
$$

and normal component of electrical displacement is

$$
D_{y}=-k \varepsilon_{0} \mathrm{e}^{k y} \mathrm{e}^{-\mathrm{i} k z}, \quad y<0 .
$$

Consequently, the unperturbed surface impedance is

$$
Z_{E}(0)=-\frac{1}{\mathrm{i} \omega k_{0} \varepsilon_{0}} .
$$

The perturbed boundary conditions will be specified in terms of the normalized surface impedance

$$
z_{E}^{\prime}(0)=\frac{Z_{E}^{\prime}(0)}{\left|Z_{E}(0)\right|}=-\mathrm{i} k_{0} \varepsilon_{0}\left(\frac{\varphi^{\prime}}{D_{y}^{\prime}}\right)_{y=0} .
$$

The perturbed fields potential $\varphi^{\prime}(0)$ and electrical displacement $D_{y}(0)$ are now related to the unperturbed fields

$$
\begin{aligned}
& \varphi^{\prime}(0)=\varphi(0)+A, \\
& D_{y}^{\prime}(0)=D_{y}(0)+k_{0} \varepsilon_{p}^{\mathrm{T}} A, \\
& \varepsilon_{p}^{\mathrm{T}}=\sqrt{\varepsilon_{y y}^{\mathrm{T}} \varepsilon_{z z}^{\mathrm{T}}-\left(\varepsilon_{y z}^{\mathrm{T}}\right)^{2}}
\end{aligned}
$$$$
\text { where }
$$

and the approximation $k_{0}^{\prime}=k_{0}$ has been made. The electrical displacement $D_{y}(0)$ and $D_{y}^{\prime}(0)$ are expressed 
in terms of potential by means of (2) and (3), and elimination of $A$ gives the electrical potential and induction, expressed in terms

$$
\begin{aligned}
& \varphi^{\prime}(0)=-\mathrm{i} z_{E}^{\prime}(0) \frac{\varepsilon_{0}+\varepsilon_{p}^{\mathrm{T}}}{\varepsilon_{0}-\mathrm{i} \varepsilon_{p}^{\mathrm{T}} z_{E}^{\prime}(0)} \varphi(0), \\
& D_{y}^{\prime}(0)=-\frac{k_{0} \varepsilon_{0}\left(\varepsilon_{0}+\varepsilon_{p}^{\mathrm{T}}\right)}{\varepsilon_{0}-\mathrm{i} \varepsilon_{p}^{\mathrm{T}} z_{E}^{\prime}(0)} \varphi(0) .
\end{aligned}
$$

By substitution of the perturbed fields (11) and (12) into (1) and use of the impedance relation (8) we obtain

$$
\frac{\Delta k}{k_{0}}=-\left(\frac{\Delta v}{v_{0}}\right)_{\text {sc }} \frac{1+\mathrm{i} z_{E}^{\prime}(0)}{1-\mathrm{i} \frac{\varepsilon_{p}^{\mathrm{T}}}{\varepsilon_{0}} z_{E}^{\prime}(0)},
$$

where the unperturbed boundary conditions are electrically free (open), and

$$
\left(\frac{\Delta v}{v_{0}}\right)_{\mathrm{sc}}=-\omega\left(\varepsilon_{0}+\varepsilon_{p}^{\mathrm{T}}\right) \frac{|\varphi(0)|^{2}}{4 P} .
$$

This is the perturbation due to an electrical short circuit on the boundary. This is the Ingebrigtsen formula for electrical surface perturbations of SAW Rayleigh waves. It is also applicable to other types of piezoelectric surface waves when the appropriate normalized surface wave potential is used in $\left(\frac{\Delta v}{v_{0}}\right)_{\mathrm{sc}}$. Since the unperturbed wave satisfies free electrical boundary conditions, then $\Delta k \rightarrow 0$ in (13) when

$$
z_{E}^{\prime}(0)=\frac{Z_{E}^{\prime}(0)}{\left|Z_{E}^{\prime}(0)\right|} \rightarrow \mathrm{i} .
$$

The influence effect between the electric potential associated with the acoustic wave and the carriers of the electric charge in this layer leads to a decrease of the velocity. This effect depends on the electromechanical coupling factor $K^{2}$. The Ingebrigtsen formula for electrical surface perturbations of SAW Rayleigh waves reduces to form $[7,8]$ :

$$
\left(\frac{\Delta k}{k_{0}}\right)=\frac{K^{2}}{2} \frac{1+\mathrm{i} z_{E}^{\prime}(0)}{1-\mathrm{i} \frac{\varepsilon_{p}^{\mathrm{T}}}{\varepsilon_{0}} z_{E}^{\prime}(0)},
$$

where

$$
K^{2}=2\left(\frac{\Delta v}{v_{0}}\right)_{\mathrm{sc}} .
$$

\subsection{Infinitesimally thin semiconducting sensor layer}

We consider first the RF interaction with an infinitesimally thin semiconductor, regarded as thinner than a Debye length. For the construction of width $w$ in the $x$ direction, the one-dimensional equation of motion is

$$
I_{z}=\rho_{01} \mu E_{z}-D \frac{\mathrm{d} \rho_{1}}{\mathrm{~d} z},
$$

where $I_{z}$ is the total RF current in the $z$ direction, $\rho_{1}$ and $\rho_{2}$ are, respectively, the RF and dc charge density per unit length. The quantities $\mu$ and $D$ are, respectively, the carrier mobility and diffusion parameters. By combining the one-dimensional equation of continuity

$$
\frac{\mathrm{d} I_{z}}{\mathrm{~d} z}+\mathrm{i} \omega \rho_{1}=0
$$

with (17) and assuming that all RF quantities vary as $\exp (\mathrm{i}(\omega t-k z))$, it follows that the RF charge field is related by

$$
\rho_{1}\left(\omega-\mathrm{i} D k^{2}\right)=k \rho_{01} \mu E_{\mathrm{Z}} .
$$

Although thin, the semiconductor layer has a finite thickness $h$, width $w$ and the volume dc charge density is

$$
\rho_{0}=\frac{\rho_{01}}{w h},
$$

where

$$
\rho_{\mathrm{s}}=\frac{\rho_{1}}{w} .
$$

Following the conventions established by White [9] for the volume acoustic wave in configurations, we define relaxation frequency:

$$
\omega_{\mathrm{c}}=\frac{\mu\left|\rho_{0}\right|}{\varepsilon}
$$

and the diffusion frequency:

$$
\omega_{\mathrm{D}}=\frac{v_{0}^{2}}{D}=\frac{k_{\mathrm{B}} T}{q} \mu,
$$

where $k_{\mathrm{B}}$ - Boltzman's constant, $T$ - temperature.

We then rewrite (19) in the form

$$
\rho_{\mathrm{s}}=\frac{\left(\frac{\omega_{\mathrm{c}}}{\omega}\right) \varepsilon k h}{1-\mathrm{i} \frac{\omega}{\omega_{\mathrm{D}}}} E_{z} .
$$

\subsection{Surface impedance of single semiconducting sensor layer}

For $y<0$ we assume isotropic conditions so that $\varepsilon$ is scalar with value $\varepsilon_{0}$. Because the piezoelectric medium is anisotropic, we take the permittivity in the region $y>$ 0 in tensor form. The appropriate value of $\varepsilon$ to use is approximately the stress-free form $\varepsilon^{\mathrm{T}}$. The piezoelectric medium (at $y>0)$ and imaginary charge sheet (at $y<0$ ) are in three regions in $y$ directions.

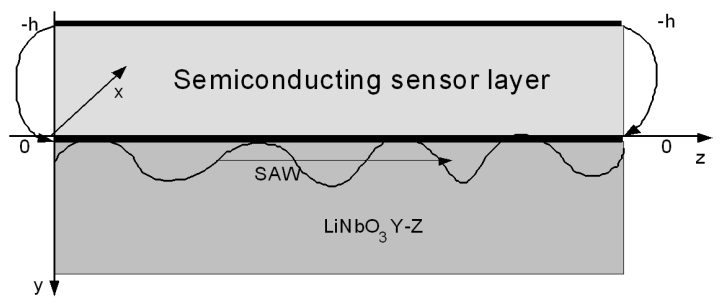

Fig. 3. Schematic diagram of the layered SAW gas sensor.

The space-charge field boundary conditions for single semiconductor layer (Fig. 3) at plain $y=0$ are respectively:

1) $\varphi\left(0^{-}\right)=\varphi\left(0^{+}\right)$,

2) $D_{y}\left(0^{-}\right)-D_{y}\left(0^{+}\right)=\rho_{\mathrm{s}}$, where

$$
D_{y}\left(0^{-}\right)=\varepsilon_{0} E_{y}\left(0^{-}\right) .
$$

Since we assume that field solution has electrostatic form $\boldsymbol{E} \rightarrow=-\nabla \varphi$, we imply that $E_{z}^{-}=\mathrm{i} k_{0} \varphi(y, z)$. 
By applying Gauss' law and potential continuity at plain $y=0$, the impedance $z_{E}^{\prime}(0)$ can be shown in the following form:

$$
z_{E}^{\prime}(0)=\left(-\mathrm{i}+\frac{\frac{\sigma_{\mathrm{s}}}{\varepsilon_{0} v_{0}}}{1-\mathrm{i} \frac{\omega}{\omega_{\mathrm{D}}}}\right)^{-1},
$$

where $\sigma_{\mathrm{s}}=\sigma h$ is the surface conductivity of the layer.

In the case when diffusion effects are small $(D \approx 0)$, surface conductivity is independent of the frequency of the wave propagation and surface impedance $z_{E}^{\prime}(0)$ is only function of the surface conductivity $\sigma_{\mathrm{s}}$ :

$$
z_{E}^{\prime}(0)=\left(-\mathrm{i}+\frac{\sigma_{\mathrm{s}}}{\varepsilon_{0} v_{0}}\right)^{-1} .
$$

\subsection{Dependence of the $S A W$ velocity vs. surface layer conductivity}

Imaginary parts in equations indicate that apart from changing velocity, there are apparent changes in the attenuation of acoustic wave. When real and imaginary parts are extracted from perturbation formula, the relative change of SAW velocity is the following:

$$
\begin{aligned}
& \frac{\Delta v}{v_{0}}=-\operatorname{Re}\left(\frac{\Delta k}{k_{0}}\right), \\
& \frac{\Delta \alpha}{k_{0}}=\operatorname{Im}\left(\frac{\Delta k}{k_{0}}\right) .
\end{aligned}
$$

When the diffusion effect vanishes $(D=0)$, the perturbation of velocity will be independent from the frequency of the propagation wave.

The reduction of the velocity of propagation is mainly caused by two phenomena $[10,11]$ :

a) The mass load on the crystal surface, resulting this time from the phenomenon of adsorption of gas particles on the layer surface, the surface density may be disturbed due to the additional mass, $\delta m$, of gas particles adsorption, and the elastic modulus of the isotropic layer, $\mu$ and $\lambda$, and

b) the electric "load" resulting from the effect of interaction of the electric potential associated with the surface wave with mobile charge carriers in the layer. This results from changes in the electric conductivity of the layer, $\sigma_{\mathrm{s}}$, due to the interaction of this chemically active layer with gas.

The electric effect has one very interesting feature, namely it causes significant changes in the propagation velocity of the SAW only in some particular range, which depends only on the properties of the piezoelectric substrate (which was $\mathrm{LiNbO}_{3}, Y-Z$ ); the metal layer shortens the electric field associated with the surface wave [5]. In spite of substantial changes in the conductivity, it does not cause any significant changes in the wave velocity, it is for the $\sigma_{\mathrm{s}} / v_{0} C_{\mathrm{s}}>5$ region. The beginning of the characteristic starts at the $\sigma_{\mathrm{s}} / v_{0} C_{\mathrm{s}}>1.5$ region.

It seems that the structure should have its resultant electrical conductivity much better fitted to the high sensitivity range in the $0.15<\sigma_{\mathrm{s}} / v_{0} C_{\mathrm{s}}<5$ region.

In the future we are planning to optimize the best structure to improve its sensitivity and response times.
According to the theory we can prove that surface conductivity has influence on the detection features of SAW gas sensors. The beginning value of the conductivity determines the point of sensor work. The value of acoustoelectric interaction influence in the sensor configurations of a piezoelectric transducer and semiconductor layer has a vital role in the sensitivity of the sensor.

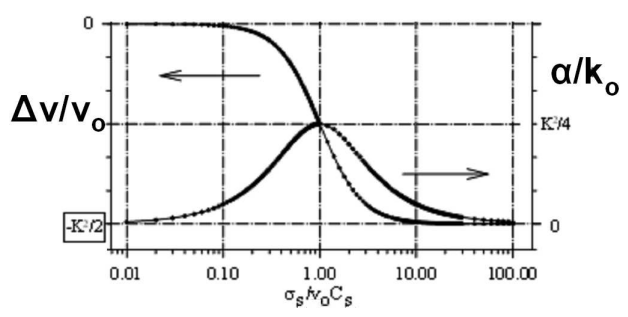

Fig. 4. Velocity of the SAW wave propagation versus electrical conductivity of the sensor layer.

In Fig. 4 a characteristic of relative change of a surface acoustic wave and its attenuation in the function of conductivity has been presented. In the case of $\mathrm{LiNbO}_{3}$, cuts $Y-Z$ as piezoelectric substrate, the biggest change in velocity is the following (the highest attenuation existing at this value) for

$$
\frac{\sigma_{\mathrm{s}}}{v_{0} C_{\mathrm{s}}}=1
$$

and from here $\sigma_{\mathrm{s}}=v_{0} C_{\mathrm{S}}=1.6 \times 10^{-6} \Omega^{-1}$.

The active range of acoustoelectric interaction (the highest change of velocity) is for

$$
0.15<\frac{\sigma_{\mathrm{s}}}{v_{0} C_{\mathrm{s}}}<5
$$

\section{Experimental verification of the two effects in SAW sensors}

The setup for verification mass effect and electric effect is presented in Fig. 5 .

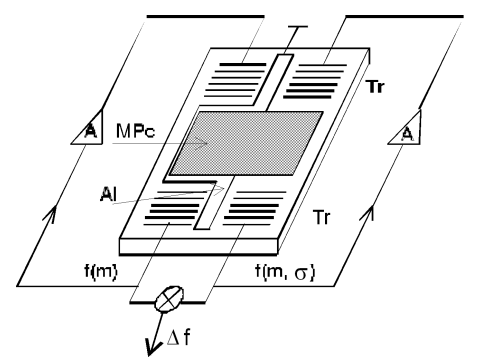

Fig. 5. Setup for experimental separating of mass and acoustoelectric effects.

To separate these effects we have been using two delay line configurations [11]. In one, the acoustic path under the sensor layer (lead phtalocyanine $\mathrm{MPc}-\mathrm{PbPc}$ ) was made by thermal evaporation process as a metallization layer (aluminum Al). This is the screen layer for the 
piezoelectric field. On both paths, by means of the thermal sublimation method, a sensor layer (MPc) is made; so in only one of the paths (without the metallization film) there is an acoustoelectric interaction (semiconductor sensor and piezoelectric field are between free charge carriers) possible. In this way the output frequency signal, $\Delta f$, is dependent on the difference between mass and electric effect. Experimental results are shown in Fig. 6 .

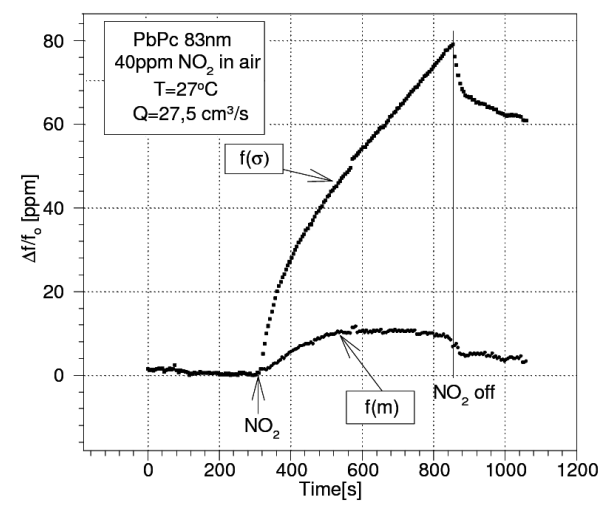

Fig. 6. Mass effect and acoustoelectric effect in SAW sensor with $\mathrm{PbPc}$ layer sensor.

\section{Conclusions}

In this paper are presented the theoretical basis of SAW gas sensors working with semiconducting thin film as a chemical sensor layer. Results which were obtained are applicable also to SAW sensors other physical quantity which uses Rayleigh waves in the piezoelectric medium.

For selective detection gas particles must be used with specific chemical sensor layers placed on acoustic line. Any changes in the physical properties of this sensor layer in gas atmosphere changes conditions for acoustic wave propagation and from this, changes in velocity propagation and the attenuation of the wave. In the paper considered separately are mass and electrical effects as perturbations in mechanical and electrical boundary conditions. Perturbations of the mechanical boundary conditions are important in a sensor with a dielectric sensor layer. Perturbations in electrical boundary conditions are important in a sensor with conducting sensor layer (or semiconducting). These effects are additive because of its small values. To separate these effects we have been using two delay line configurations. Experimental results have been shown.

\section{Acknowledgments}

This work is financed as the grant of Ministry of Science and Higher Education No. N N505 374237.

\section{References}

[1] B.A. Auld, Acoustic Fields and Waves, Vol. 2, Wiley, New York 1973.

[2] G.S. Kino, IEEE Trans. Electron Dev. ED-18, 909 (1971).

[3] W. Jakubik, M. Urbańczyk, Arch. Acoust. 22, 187 (1997).

[4] W. Jakubik, Thin Solid Films 515, 8345 (2007).

[5] W. Jakubik, Sensors Actuators B 96, 321 (2003).

[6] T. Pustelny, A. Opilski, B. Pustelny, Acta Phys. Pol. A 114, A-181 (2009).

[7] W. Jakubik, M. Urbańczyk, E. Maciak, T. Pustelny, Acta Phys. Pol. A 116, 315 (2009).

[8] W. Jakubik, M. Urbańczyk, J. Tech. Phys. IV 38, 589 (1997).

[9] D.L. White, J. Appl. Phys. 33, 2547 (1962).

[10] B. Pustelny, T. Pustelny, Acta Phys. Pol. A 116, 383 (2009).

[11] M. Urbańczyk, Arch. Acoust. 22, 187 (1997). 